\title{
Neurokinin-1 Receptor (NK1-R) Expression in the Brains of SIV-Infected Rhesus Macaques
}

\author{
Implications for Substance P in NK1-R Immune Cell \\ Trafficking into the CNS
}

Heather Vinet-Oliphant, ${ }^{\star \dagger}$ Xavier Alvarez, ${ }^{*}$ Elizabeth Buza, ${ }^{\ddagger}$ Juan T. Borda, ${ }^{*}$ Mahesh Mohan, ${ }^{*}$ Pyone P. Aye, ${ }^{*}$ Florin Tuluc, ${ }^{\S}$ Steven D. Douglas, ${ }^{\S}$ and Andrew A. Lackner*

From the Division of Comparative Pathology,* Tulane National Primate Research Center, Covington, Louisiana; the Department of Biomedical Engineering, ${ }^{\dagger}$ School of Science and Engineering, Tulane University, New Orleans, Louisiana; School of Veterinary Medicine, ${ }^{\ddagger}$ Louisiana State University, Baton Rouge, Louisiana; and the Division of Allergy and Immunology, the Department of Pediatrics, ${ }^{\S}$ University of Pennsylvania School of Medicine and the Children's Hospital of Philadelphia Research Institute, Children's Hospital of Philadelphia, Philadelphia, Pennsylvania

Recent studies suggest a link between neuropsychiatric disorders and HIV/SIV infection. Most evidence indicates that monocytes/macrophages are the primary cell type infected within the CNS and that they contribute to CNS inflammation and neurological disease. Substance P (SP), a pleotropic neuropeptide implicated in inflammation, depression, and immune modulation via interaction with its cognate receptor, the neurokinin 1 receptor (NK1-R), is produced by monocyte/macrophages. While the presence of NK1-R on neurons is well known, its role on cells of the immune system such as monocyte/macrophages is just beginning to emerge. Therefore, we have examined the expression of SP and NK1-R and their relationship to SIV/HIV encephalitis (SIVE/HIVE) lesions and SIV-infected cells. These studies demonstrated intense expression of SP and NK1-R in SIVE lesions, with macrophages being the principal cell expressing NK1-R. Interestingly, all of the SIV-infected macrophages expressed NK1-R. Additionally, we examined the functional role of SP as a proinflammatory mediator of monocyte activation and chemotaxis. These studies demonstrated that treatment of monocytes with SP elicited changes in cell-surface expression for CCR5 and NK1-R in a dose-dependent manner. Moreover, pretreatment with SP enhanced both SP- and CCL5-mediated chemotaxis. All of these findings suggest that SP and NK1-R are important in SIV infection of macrophages and the development of SIVE lesions. (Am J Pathol 2010, 177:1286-1297; DOI: 10.2353/ajpath.2010.091109)

The neuropathogenesis of HIV infection is complex and has led to the development of nonhuman primate models using infection of macaques with the simian immunodeficiency virus (SIV). The human and simian immunodeficiency viruses are closely related and produce nearly identical conditions in their respective hosts, thus the rhesus macaque infected with SIV is the premier animal model for the study of AIDS pathogenesis in general and of the neuropathogenesis of AIDS in particular. Additionally, nonhuman primates are widely used in neuroscience research, including neurophysiologic and neurobehavioral studies. Recent studies suggest a link between psychiatric disorders like stress and depression and the neuropathogenesis of HIV and/or its progression to AIDS. ${ }^{1,2}$ Neuroinvasion by SIV and HIV occurs early in infection at the time of peak viremia. The virus most likely enters the brain within cells of monocyte/macrophage lineage, and the perivascular macrophage is the primary cell type productively infected within the CNS. ${ }^{3,4}$

Approximately 25 to $30 \%$ of untreated HIV-infected adult humans develop a debilitating neurological disor-

Supported in part by Public Health Service grants RR000164, RR016930, RR018397, NS30769, and MH076388.

Accepted for publication April 29, 2010.

Supplemental material for this article can be found on http://ajp. amjpathol.org

Address reprint requests to Andrew A. Lackner, D.V.M., Ph.D., Tulane National Primate Research Center, Division of Comparative Pathology, Covington, LA 70433. E-mail: alackner@tulane.edu. 
der termed AIDS dementia complex (ADC). ${ }^{5-7} \mathrm{~A}$ wide variety of cytokines, which include IL-1beta, IL-6, and TNF- $\alpha$, chemokines (CCL2, CCL3, CCL4 and CCL5), adhesion molecules, and other molecules such as Substance $\mathrm{P}(\mathrm{SP})$ produced by different cell types have been implicated in this process. ${ }^{1,4,8,9}$ Microscopic evaluation of brains from individuals with ADC reveals a broad spectrum of pathological features including neuronal changes, multifocal encephalitis, accumulation of inflammatory macrophages and multinucleated giant cells (MNGCs), cerebral cortical atrophy, and white matter pallor. ${ }^{5,10}$ The histopathological substrate of ADC, referred to as HIV encephalitis (HIVE), is characterized by perivascular accumulation of macrophages and MNGCs in the CNS with abundant infection of brain macrophages. ${ }^{4,11}$ In addition to the pathological manifestations within the brains of individuals with ADC, extensive neurobehavioral effects have been characterized and include impaired fine motor control and memory, altered emotional control, motor slowing, and possibly depression. Compelling data from recent studies reveal the significance of inflammatory mediators including the neuropeptide SP in the neuropathogenesis of AIDS ${ }^{12-14}$ as well as in clinical neurobehavioral effects of depression. ${ }^{2-4}$ Such findings suggest that SP participates in important aspects of immune-neural communication and likely contributes to immune modulation in HIV/SIV infection.

$\mathrm{SP}$ is an 11-aa neuropeptide and is the most extensively studied and most abundant member of the tachykinin family. SP is synthesized mainly by primary sensory neurons, however recent studies have demonstrated that immune cells also express SP mRNA and protein during SIV/HIV-infection, ${ }^{1,8}$ suggesting that it may contribute to neurological disease by acting on its preferred receptor, NK1-R, a G-protein-coupled receptor expressed on Tcells, B-cells, monocyte/macrophages, NK cells, astrocytes, and neurons. ${ }^{9}$ Activation of NK1-R by SP results in increased phagocytic response in macrophages, enhanced inflammatory cytokine production by immune cells, and possible induction of a chemotactic response in monocyte macrophages, thus facilitating immune cell trafficking at sites of inflammation or infection. ${ }^{16-20} \mathrm{SP}$ has a role in AIDS, and results from recent in vivo studies $1,8,15,21,22$ revealed that NK1-R antagonists have an antiviral effect, ${ }^{23}$ likely through down-regulation of CCR5 ${ }^{9}$ as well as immunomodulatory and antidepressive effects. ${ }^{23,24}$ The major focus of existing work on SP (and its receptor-NK1-R) in macaques, however, has focused on its role as a neurotransmitter or neuromodulator and therefore the distribution of SP and NK1-R on neurons is well known, while little data exists on the in vivo distribution of SP and NK1-R on other cell types.

Because the cellular expression of SP and its receptor NK1-R is not fully characterized in normal or SIV-infected macaques, we examined the cellular distribution/location and phenotype of SP and NK1-R expressing cells in the CNS in vitro and in vivo using multiple techniques and at various stages of infection in animals with or without SIVE. Such analysis allowed us to assess spatial correlations between the presence of SIV-infected cells and cells positive for SP and NK1-R. Additionally, we examined the functional aspects of SP/NK1-R signaling in monocyte activation and chemotaxis. Data from our immunofluorescence and in situ hybridization studies show that while NK1-R is expressed in astrocytes and neurons, it is intensely expressed in SIVE lesions. NK1-R expression was detected in all SIV-infected cells associated with SIVE lesions, of which macrophages were the predominant cell type and also the primary cell type expressing NK1-R. T-cells were found in small numbers in SIVE lesions and were rarely infected but were shown to express NK1-R when present. Our quantitative flow cytometric analysis demonstrates that SP functionally regulates NK1-R and CCR5 expression on macaque monocytes. Furthermore, pretreatment of monocytes with SP enhanced SP-mediated chemotaxis as well as CCL5mediated chemotaxis. The enhancement of SP-mediated chemotaxis in the presence of CCL5 demonstrates cross talk between NK1-R and CCR5 signaling pathways. These findings suggest that SP contributes to SIV-associated neurological disease and suggest that SP also has a role in augmented cellular trafficking across the bloodbrain barrier and thus the development of SIVE lesions. This insight into the function of SP and NK1-R allows for a better understanding of the interaction between the immune and nervous systems, and may lead to advancements in treatment of neurological and neuropsychiatric disease.

\section{Materials and Methods}

\section{Animals, Tissues, and Virus}

Tissues from 20 SIV-infected and two uninfected Indianorigin rhesus macaques (Macaca mulatta) obtained from the pathology archives of the Tulane National Primate Research Center were used for these studies (Table 1). Eight of the animals were from a time course study, of which two animals were euthanized at 7, 14, 21, and 50 days after inoculation. The remaining 12 animals were all euthanized with terminal AIDS. Eight of the 12 animals with terminal AIDS had SIVE, while four did not. Details on these animals, including time after inoculation and major pathological findings, are provided in Table 1 and have been described previously. ${ }^{25}$ All animals were infected intravenously with $50 \mathrm{ng}$ p27 of virus and housed and maintained in accordance with the standards of the American Association for Accreditation of Laboratory Animal Care and the "Guide for the Care and Use of Laboratory Animals" prepared by the National Research Council. All studies were approved by the Tulane Institutional Animal Care and Use Committee.

When moribund with AIDS or following the time course study, animals were anesthetized using Ketamine $\mathrm{HCL}$ and humanely euthanized with an intravenous overdose of pentobarbital and were immediately necropsied. A complete set of tissues including multiple levels of brain were collected for formalin fixation, and paraformaldehyde fixation followed by sucrose protection and snap freezing. Tissues fixed in 10\% neutral buffered formalin were embedded in paraffin and cut into 5- to 6 - $\mu \mathrm{m}$-thick 
Table 1. Animals, Inoculum, Time of Tissue Collection, and Major Pathological Findings

\begin{tabular}{cclc}
\hline Animal No. & Days PI & \multicolumn{1}{c}{ Major pathologic findings } & Inoculum \\
\hline $250-93$ & 7 & End of experiment, NSL & SIVmac239 \\
$277-93$ & 7 & End of experiment, NSL & SIVmac239 \\
$285-93$ & 14 & End of experiment, NSL & SIVmac239 \\
$388-93$ & 14 & End of experiment, NSL & SIVmac239 \\
$406-93$ & 21 & End of experiment, NSL & SIVmac239 \\
$441-93$ & 21 & End of experiment, NSL & SIVmac239 \\
$364-93$ & 50 & End of experiment, NSL & SIVmac239 \\
$434-93$ & 50 & End of experiment, NSL & SIVmac239 \\
AH88 & 134 & SIV, arteritis & SIVmac251 \\
BI63 & 150 & SIV, thrombosis & SIVmac251 \\
DD88 & 383 & SIV, disseminated M. avium complex & SIVmac239 \\
AT71 & 121 & SIVE, giant cell pneumonia & SIVmac251 \\
G402 & 86 & SIVE, giant cell pneumonia & SIVmac239 \\
AH01 & 93 & SIVE, giant cell pneumonia, gastric thrichomoniasis, & SIVmac239 \\
AM93 & 136 & SIVE, giant cell pneumonia & SHIV162 + 33A \\
V791 & 199 & SIVE, optic neuritis, giant cell pneumonia & Yes \\
FA97 & 316 & SIVE, meningoencephalitis & SIVmac251 \\
P114 & 1807 & SIVE, gliosis & SIVmac239 \\
T682 & 680 & SIVE, meningoencephalitis & YHIV33 \\
BR06 & 177 & SIVE & YIVdeltaB670 \\
DI95 & n/a & Colitis & SIVmac251 \\
BV36 & n/a & Colitis & Uninfected \\
\hline
\end{tabular}

Days PI indicates days after inoculation; NSL, no significant lesions; SIVE, SIV encephalitis.

sections and placed on slides. Adjacent tissues were fixed in $2 \%$ paraformaldehyde for four hours at room temperature. Paraformaldehyde-fixed tissues were then washed three times in PBS and placed in 30\% buffered sucrose overnight. Following sucrose protection, tissues were embedded in optimal cutting temperature compound OCT (Tissue Tek, Torrance CA) and snap frozen in 2-methylbutane cooled by dry ice and cut into sections 5-8 $\mu \mathrm{m}$ thick and placed on slides.

\section{Immunohistochemistry}

Deparaffinized sections were assessed by immunohistochemistry for SP using a monoclonal antibody made in rat (Sigma Chemical Co., St. Louis, MO) (Table 2). Immunohistochemistry was performed using avidin-biotin peroxidase complex (Vectastain kit; Vector Laboratories, Burlingame, CA) or peroxidase-conjugated polymer reagent (SuperPicTure kit; Zymed, San Francisco, CA) according to the manufacturer's instructions. Before primary antibody incubation, serum or nonserum protein block was applied for 2 hours at room temperature. The color reaction product was developed using 3,3'-diaminobenzidine tetrahydrochloride (DAB; DAKO, Carpinteria, CA) as the chromogenic substrate for horseradish peroxidase. The sections were then dehydrated and mounted. Controls consisted of matched tissues, omission of the primary antibody, and substitution of an irrelevant isotypematched immunoglobulin at the same concentration. The sections were mounted using Faramout aqueous mounting medium (DAKO).

\section{Localization of SIV-Infected Cells}

In situ hybridization for SIV RNA was performed using SIV-specific riboprobes as previously described. ${ }^{25}$ Following deparaffinization, tissue sections were pretreated in a microwave with citrate buffer (antigen unmasking solution; Vector Laboratories, Burlingame, CA) for 20 minutes at high power. Thereafter, sections were thoroughly washed with $2 \times$ standard saline citrate, placed in a RNase free humidified chamber, and prehybridized at $45^{\circ} \mathrm{C}$ with hybridization buffer (containing $50 \%$ formamide, 20\% 20× standard saline citrate (Roche, Penzberg, Germany), 22\% DEPC $\mathrm{H}_{2} \mathrm{O}$ (Sigma), 0.4\% 0.5M EDTA (Sigma), 2\% 50× Denhardt's solution (Sigma), and

Table 2. Antibodies Used in Immunohistochemistry (IHC) and Immunofluorescence (IF)

\begin{tabular}{llllll}
\hline \multicolumn{1}{c}{ Cell type } & Antigen & Source & Clone & & Isotype* \\
\hline Astrocyte & GFAP & Sigma & GA5 & IgG1 & Dilution \\
Astrocyte & GFAP & Sigma & GA5 & IgG1 conjugated with CY3 & $1: 200$ \\
Macrophage & CD68 & DAKO & Kp1 & IgG1 & $1: 500$ \\
Macrophage & CD163 & Serotec & EDHu-1 & IgG1 & $1: 50$ \\
Macrophage & HAM56 & DAKO & HAM56 & IgM & $1: 20$ \\
Neuron & NEU-N & Chemicon & a60 & IgG1 & $1: 10$ \\
Neuron & NSE & DAKO & 5E2 & IgG2a & $1: 5$ \\
NK1-R-expressing cells & NK1-R & Sigma & & Polyclonal & $1: 50$ \\
SP producing cells & SP & Sigma & & Polyclonal & $1: 50$ \\
T lymphocyte & CD3 & DAKO & F72.38 & IgG1 & $1: 20$ \\
\hline
\end{tabular}

${ }^{*}$ All antibodies are murine monoclonals except polyclonal antibodies to NK1-R and SP, which were made in rabbit and rat, respectively. 
Table 3. Antibody and Fluorochrome Combinations for Triple-Label Confocal Microscopy

\begin{tabular}{|c|c|c|c|c|c|}
\hline \multicolumn{2}{|c|}{ Channel 1 red with Permanent Red or Alexa 568} & \multicolumn{2}{|c|}{$\begin{array}{c}\text { Channel } 2 \text { green with } \\
\text { Alexa } 488\end{array}$} & \multicolumn{2}{|c|}{ Channel 3 far-red with Alexa 633} \\
\hline $\begin{array}{l}\text { Riboprobes } \\
\text { Riboprobes } \\
\text { Riboprobes } \\
\text { CD3 (IgG1) mouse } \\
\text { CD163 (IgG1) mouse } \\
\text { GFAP (IgG1) mouse (CY3) } \\
\text { CD3 (IgG1) mouse } \\
\text { NSE (IgG2a) mouse }\end{array}$ & $\begin{array}{l}\text { Anti-digoxigenin/sheep } \\
\text { Anti-digoxigenin/sheep } \\
\text { Anti-digoxigenin/sheep } \\
\text { Anti-rabbit } \\
\text { Anti-mouse (IgG1) } \\
\text { Anti-mouse (lgG1) } \\
\text { Anti-mouse (IgG2a) }\end{array}$ & $\begin{array}{l}\text { NK1-R (rabbit) } \\
\text { NK1-R (rabbit) } \\
\text { NK1-R (rabbit) } \\
\text { NK1-R (rabbit) } \\
\text { NK1-R (rabbit) } \\
\text { NK1-R (rabbit) } \\
\text { NK1-R (rabbit) } \\
\text { NK1-R (rabbit) }\end{array}$ & $\begin{array}{l}\text { Anti-rabbit } \\
\text { Anti-rabbit } \\
\text { Anti-rabbit } \\
\text { Anti-rabbit } \\
\text { Anti-rabbit } \\
\text { Anti-rabbit } \\
\text { Anti-rabbit } \\
\text { Anti-rabbit }\end{array}$ & $\begin{array}{l}\text { GFAP (IgG1) mouse } \\
\text { CD163 (lgG1) mouse } \\
\text { CD3 (IgG1) mouse } \\
\text { Nuclear Staining ToPro3 } \\
\text { Nuclear Staining ToPro3 } \\
\text { NSE (IgG2a) mouse } \\
\text { HAM56 (IgM) mouse } \\
\text { GFAP (IgG1) mouse }\end{array}$ & $\begin{array}{l}\text { Anti-mouse (IgG1) } \\
\text { Anti-mouse (IgG1) } \\
\text { Anti-mouse (IgG1) }\end{array}$ \\
\hline
\end{tabular}

Each column shows the primary and secondary antibody (if relevant) used. Channel 1 was used for i) visualization of in situ hybridization using Permanent Red, which fluoresces intensely red; ii) primary antibodies conjugated to CY3; or iii) secondary antibodies conjugated with Alexa 568 . Channel 2 was used to detect NK1-R using secondary antibody conjugated to Alexa 488, and Channel 3 was used to detect additional labels using secondary antibodies conjugated to Alexa 633. The color of the far red channel is changed to blue at the time of data acquisition on the confocal microscope.

dextran sulfate (Sigma) at $100 \mathrm{mg} / \mathrm{ml}$ with denatured herring sperm DNA and yeast tRNA at $10 \mathrm{mg} / \mathrm{ml}$ each) for 1 hour. Following prehybridization incubation, sections were hybridized overnight at $45^{\circ} \mathrm{C}$ with SIV-digoxigeninlabeled antisense riboprobes (Lofstrand Laboratories, Gaithersburg, MD) at a concentration of $10 \mathrm{ng} / \mathrm{slide}$ in hybridization buffer. Following hybridization, slides were washed with $2 \times$ standard saline citrate, $1 \times$ standard saline citrate, and $0.1 \times$ standard saline citrate diluted in DEPC $\mathrm{H}_{2} \mathrm{O}$ at $45^{\circ} \mathrm{C}$. Slides were then washed in $1 \times$ Tris buffered saline (TBS; DAKO) at room temperature and incubated with a $1 \times$ in situ blocking solution (Vector Laboratories) in a humidified chamber for thirty minutes at room temperature. Fab fragments of an anti-digoxigenin antibody from sheep, conjugated with alkaline phosphatase (Roche, Penzberg, Germany) were used to detect digoxigenin labeled probes. Controls included matched tissues from known positives and negatives and hybridization with digoxigenin-labeled sense RNA labeled with digoxigenin.

When immunofluorescence followed in situ hybridization, the liquid permanent red substrate-chromogen detection system using alkaline phosphatase (Dako) was used. Briefly, slides were rinsed in $1 \times$ Tris-buffered saline, and then $200 \mu \mathrm{l} / \mathrm{slide}$ of liquid permanent red Substrate Buffer-Chromogen reagent ( $1 \mathrm{ml}$ of Liquid Permanent Red Chromogen in $30 \mathrm{ml}$ of Liquid Permanent Red Substrate Buffer) was applied. The slides were incubated for 30 minutes at room temperature in the dark. Once developed the slides were thoroughly rinsed with $1 \times$ Tris-buffered saline solution and fixed with $2 \%$ paraformaldehyde for 7 minutes. The slides were then blocked with a $100 \mathrm{mmol} / \mathrm{L}$ Glycine solution diluted in $1 \times$ PBS for an additional 7 minutes and stored in $1 \times$ TBS until stained using immunofluorescence techniques.

\section{Immunofluorescence for NK1-R and Cell Phenotype}

Triple label immunofluorescence detection to examine the cellular distribution of NK1-R in the CNS of normal and SIV-infected macaques at various stages of diseases was performed to determine the relationship among SP, NK1-R, and CNS lesions using previously described techniques. ${ }^{25}$ Single-label immunofluorescence detec- tion for NK1-R was initially performed using a polyclonal rabbit antibody. Details of the antibodies used in this study are given in Table 2, and combinations of antibodies and fluorochromes are provided in Table 3. Paraformaldehyde fixed frozen sections were initially washed in phosphate-buffered saline containing $0.2 \%$ fish skin gelatin and $0.1 \%$ triton-X100 (PBS-FSG-Tx100) for 30 minutes at room temperature to remove OCT. Deparaffinized sections or paraformaldehyde-fixed frozen sections were then washed with phosphate-buffered saline containing $0.2 \%$ fish skin gelatin (PBS-FSG) at room temperature and blocked with $10 \%$ normal goat serum (NGS) diluted in PBS-FSG in a humidified chamber also at room temperature for 40 minutes. Primary antibodies were diluted in blocking solution (NGS) and incubated for 1 hour at room temperature in a humidified chamber protected from light. Following incubation the slides were washed twice with PBS-FSG-Tx100 for 10 minutes each. NK1-R immunofluorescence was revealed using species-specific goat anti-rabbit secondary antibodies coupled with Alexa 488 (green) or Alexa 568 (red) (Molecular Probes, Eugene, OR). Secondary antibodies were also diluted in 10\% NGS and incubated in a humidified chamber for 40 minutes at room temperature protected from light. Following incubation, the slides were washed twice with PBS-FSG-Tx100 for 10 minutes each and followed with PBS-FSG.

Additional double- or triple-label immunofluorescence was then performed sequentially to determine the immunophenotype of cells expressing NK1-R using combinations of antibodies as shown in Table 3. Several cell type-specific antibodies were used to define specific cell phenotypes including CD163, CD68, and HAM56 for macrophages; CD3 for T-cells; NeuN (neuronal nuclei) and NSE (neuron specific enolase) for neurons; and GFAP (glial fibrillary acidic protein) for astrocytes (Table 2). Immunofluorescence detection of these cell types was performed as described above following blocking with 10\% NGS. The cell type-specific antibodies CD163, CD68, HAM56, CD3, NeuN, NSE, and GFAP were visualized with isotype-specific goat anti-mouse secondary antibodies labeled with either Alexa 568 (red) or Alexa 633 (far red) (Molecular Probes). On completion of immunofluorescence staining, the sections were rinsed in doubly distilled water and mounted in an anti-quenching 
solution ( $0.33 \mathrm{~g} / \mathrm{ml}$ Glycerol (analar grade), $0.133 \mathrm{~g} / \mathrm{ml}$ MOWIOL 4-88 (Calbiochem), 33.33\% double distilled water, 66.66\% 0.2M Tris Buffer, and $25 \mathrm{mg} / \mathrm{ml}$ DABCO (1.4-diazobicyclo-[2.2.2.]-octane) (Sigma) and coverslipped. Controls consisted of matched tissues and substitution of an irrelevant isotype-matched immunoglobulin at the same concentration for each primary antibody.

\section{Colocalization of Infected Cells and Cells Expressing NK1-R}

To examine the possible spatial correlations between the presence of SIV-infected cells and cells expressing NK1-R we performed combined in situ hybridization/immunofluorescence. After the results of the in situ hybridization for SIV were visualized with Permanent Red, which fluoresces intensely red, single label immunofluorescence was performed using a polyclonal rabbit NK1-R specific antibody (Table 2). After development of in situ hybridization with the permanent red substrate system and fixation and blocking as described above, the sections were washed with PBS-FSG at room temperature and blocked with 10\% NGS diluted in PBS-FSG for 40 minutes at room temperature in a humidified chamber protected from light. NK1-R immunofluorescence was performed as described above and visualized with goat anti-rabbit specific secondary antibodies coupled with Alexa 488 (green) (Molecular Probes) also as described above. Additional labeling was then performed sequentially to determine the immunophenotype of infected cells and cells expressing NK1-R as described above starting with blocking with 10\% NGS. Several cell type-specific antibodies were used in varying combinations as shown in Table 3 to define specific cell phenotypes including CD163, CD68, and HAM56 for macrophages; CD3 for T-cells; NeuN (neuronal nuclei) and NSE for neurons; and GFAP (glial fibrillary acidic protein) for astrocytes. The cell type-specific antibodies were detected with isotype specific goat anti-mouse secondary antibodies conjugated with Alexa Fluor 633 (far red) or Alexa 568 (red) (Molecular Probes). On completion of immunofluorescence staining, sections were mounted and coverslipped as described above. Controls consisted of matched tissues and substitution of an irrelevant isotype-matched immunoglobulin at the same concentration for each primary antibody.

\section{Confocal Microscopy}

Confocal microscopy was performed using a Leica TCS SP2 confocal microscope equipped with three lasers (Leica Microsystems, Exton, PA). Thirty-two to 62 optical slices were collected at $512 \times 512$ pixel resolution. Each individual slice represented $0.2 \mu \mathrm{m}$. The fluorescence of individual fluorochromes was captured separately in sequential mode after optimization to reduce bleed-through between channels (photomultiplier tubes) using Leica software. NIH Image version 1.62 and Adobe Photoshop version 7.0 were used to assign correct colors of up to four channels collected, including the fluoro- chromes Alexa Fluor 488 (green), Alexa Fluor 568 (red), Alexa Fluor 633 (far red/blue), and the differential interference contrast image (DIC) (gray scale). In some tissues and to differentiate between individual cells, To-Pro3 (nuclear marker, Molecular Probes) was used at $1 \mu \mathrm{g} / \mathrm{ml}$, incubated for 10 minutes, and tissues were washed in PBS. Colocalization of antigens is demonstrated by the addition of colors as indicated in the figure legends.

\section{Isolation of Monocytes}

Monocytes were isolated from heparinized venous blood obtained from four normal and 12 SIV-infected rhesus macaques as described previously by Chernova et al. ${ }^{26}$ In brief, peripheral blood mononuclear cells (PBMCs) were isolated from heparinized blood by centrifugation over lymphocyte separation medium (MP Biomedicals, Aurora, $\mathrm{OH})$. The mononuclear cell layer was collected and resuspended in DMEM/F12 (Gibco) completed with $5 \%$ fetal bovine serum (FBS; Gibco) and 1\% Penicillin/ Streptomycin mixture (Gibco) (DMEM/F12 Complete) and plated onto Collagen I-coated culture flasks and incubated for 45 minutes in $5 \% \mathrm{CO}_{2}$ at $37^{\circ} \mathrm{C}$ to allow for monocyte adherence. Following incubation, the flasks were washed with DMEM/F12 Complete to remove nonadherent lymphocytes, and adherent monocytes were then detached by incubation with $0.5 \mathrm{M}$ EDTA (Sigma) in DMEM/F12 containing 20\% FBS (Gibco) and 1\% Penn/ Strep mixture (Gibco). The detached cells were then cultured in suspension for 24 hours in $5 \% \mathrm{CO}_{2}$ at $37^{\circ} \mathrm{C}$ in DMEM/F12 Complete before stimulation experiments to allow for up-regulation of CCR5 and other cell surface receptors as described by Chernova et al. ${ }^{26}$

\section{Flow Cytometry}

To confirm expression of NK1-R and CCR5 on circulating rhesus macaque leukocytes, fresh $\mathrm{Na}$-heparin whole blood was collected from eight normal and four SIVinfected rhesus macaques and examined for leukocyte subsets, with a focus on monocyte/macrophages and T-cells, in addition to cellular expression of NK1-R and CCR5. Cells were stained for four-color flow cytometric analysis using the panel of markers (see Supplemental Table 1 at $h$ ttp://ajp.amjpathol.org). Briefly, PBMCs were isolated from heparinized blood by centrifugation over lymphocyte separation medium (MP Biomedicals) and resuspended in $200 \mu \mathrm{l}$ PBS. Cells were stained by incubating with the NK1-R primary antibody at $4^{\circ} \mathrm{C}$ for 30 minutes. Following incubation with the primary antibody, cells were washed with PBS, centrifuged, and resuspended in $200 \mu$ l PBS. The NK1-R primary antibody was then detected with a goat anti-rabbit secondary antibody conjugated with either AlexaFluor 488 or AlexaFluor 633 (Molecular Probes) for 30 minutes at $4^{\circ} \mathrm{C}$. Following incubation, cells were again washed with PBS, centrifuged, and resuspended in $200 \mu$ l PBS. The cells were stained with the remaining conjugated antibodies by incubation for 30 minutes at $4^{\circ} \mathrm{C}$. Cells were then washed with PBS 
and resuspended in BD Stabilizing Fixative (Becton Dickenson).

Flow cytometric analysis was also used in subsequent monocyte stimulation experiments, described below, to analyze expression of NK1-R and CCR5 in response to treatment with various concentrations of SP. For such experiments, following stimulation, the isolated monocytes were collected from the culture plates and washed and resuspended in PBS. Monocytes were then stained for flow cytometric analysis as described above. For each experiment, controls consisted of appropriate unstained and irrelevant isotype labeled samples as well as single channel samples to verify the staining specificity of the antibodies and to ensure appropriate compensation of fluorochromes during data acquisition. Data were acquired on a LSRII Flow Cytometer (Becton Dickinson) and analyzed with FlowJo v7.1 software (Tree Star, Ashland, OR).

\section{PBMC Culture to Assess Functional NK1-R Expression}

The regulation of NK1-R expression was assessed in vitro using freshly isolated rhesus macaque monocytes. The isolated monocytes were then suspended in DMEM/F12 Complete with $0.2 \%$ bovine serum albumin (BSA; Sigma) at a concentration of $2 \times 10^{6} \mathrm{cells} / \mathrm{ml}$ and treated in suspension with either a control buffer solution (DMEM/ F12 Complete $w / 0.2 \%$ BSA) or varying concentrations of SP ([Sar $\left.{ }^{9}, \operatorname{Met}\left(\mathrm{O}_{2}\right)^{11}\right]$-Substance P; Sigma) as indicated in the figure legends for 30 minutes with $5 \% \mathrm{CO}_{2}$ at $37^{\circ} \mathrm{C}$. Following treatment, the media was decanted and the cells were washed with PBS, and flow cytometry for CD3, CD14, CCR5, and NK1-R (see Supplemental Table 1 at http://ajp.amjpathol.org) was performed to assess changes in cell receptor expression on $T$ cells and monocyte/macrophages.

\section{Migration Assay to Assess Chemotactic Capacity of Substance $P$}

A transmigration assay was performed to assess the chemotactic capacity of SP on macaque monocytes. Additionally, the chemotactic capacity of SP was correlated to that of CCL5 as well as to the combination of SP and CCL5 to determine possible potentiating effects of CCL5 on SP-induced chemotaxis. The monocytes isolated from heparinized venous blood of 8 SIV-infected rhesus macaques were pretreated with control buffer (DMEM/F12 Complete w/0.2\% BSA) or $10^{-7} \mathrm{M} \mathrm{SP}\left(\left[\mathrm{Sar}^{9}, \operatorname{Met}\left(\mathrm{O}_{2}\right)^{11}\right]\right.$ Substance P; Sigma) for 30 minutes with $5 \% \mathrm{CO}_{2}$ at $37^{\circ} \mathrm{C}$. Following pretreatment with either media or SP, the monocytes were placed in the upper compartment of a 24-well transwell migration chamber (Sigma) at a concentration of approximately $2 \times 10^{5} \mathrm{cells} / \mathrm{ml}$. A filter with a 5- $\mu \mathrm{m}$ pore size, the optimal size for monocyte/macrophage cell migration, separated the upper and lower compartments of the transwell chamber. The lower compartment of the chamber contained one of four chemoattractant media; i) control buffer (DMEM/F12 Complete w/0.2\% BSA), ii) $10^{-7} \mathrm{M} \mathrm{SP}$, iii) $10 \mathrm{ng} / \mathrm{ml}$ rhCCL5 (clone 300-06; PeproTech, Rocky Hill, NJ), or iv) $10^{-7}$ M SP plus $10 \mathrm{ng} / \mathrm{ml} \mathrm{CCL5}$, all of which were diluted in the original buffer solution (DMEM/F12 Complete w/0.2\% BSA). The cells were allowed to migrate through the filter for 2 hours in an incubator at $37^{\circ} \mathrm{C}$ with $5 \% \mathrm{CO}_{2}$. Following incubation, the media remaining in the upper chamber was aspirated and 0.5 M EDTA (Sigma) in DMEM/F12 containing 20\% FBS (Gibco), and 1\% Penn/Strep mixture (Gibco) was added to the upper compartment to promote detachment of monocytes remaining within the filter. Once adherent cells were detached from the filter, these cells were added to the suspension of migrated cells and allowed to adhere to the culture plate for at least 45 minutes at $37^{\circ} \mathrm{C}$ with $5 \% \mathrm{CO}_{2}$. Once adhered, the total number of migrated cells (range of 3000 to 34,000 ) for each animal in each experimental group was determined by microscopic examination.

\section{Statistical Analysis}

Cell receptor expression for NK1-R and CCR5 among treatment groups in the monocyte culture experiments was compared using the Kruskal-Wallis test. When the results of Kruskal-Wallis test indicated a significant difference at the $P<0.05$ level between the groups examined, Tukey's multiple comparison test was then used to identify the groups that differed at the $P<0.05$ significance level. Wilcoxon Signed Ranks test was used to determine whether there were an increased number of migrated monocytes in response to the chemotactic stimuli of SP, CCL5, or SP and CCL5 compared with that of media alone for both pretreatment groups in the chemotaxis assay. Monocyte migration (\% increase) among treatment groups in the chemotaxis assay were also compared using the Kruskal-Wallis test. When the results of Kruskal-Wallis test indicated a significant difference at the $P<0.05$ level between the groups examined, Tukey's multiple comparison test was then used to identify the treatment groups that differed at the $P<0.05$ significance level. The Wilcoxon Signed Ranks test was also used to determine whether there was an effect of pretreatment with SP on monocyte migration (\% increase) for each chemotactic media.

\section{Results}

\section{Localization of Substance P Expression in SIVE}

Localization of SP expression in brains of normal, SIVinfected, and SIV-infected animals with SIVE was examined by immunohistochemistry. In the CNS of normal and SIV-infected macaques without SIVE, expression of SP was limited to neurons and astrocytes primarily in the gray matter (see Supplemental Figure S1 at http:// amjpathol.org) as described. ${ }^{27,28}$ In brains of macaques with SIVE however, intense expression of SP was associated with SIVE lesions in the white matter (Figure 1, A and B). Within SIVE lesions SP immunoreactivity was detected mainly in cells that were morphologically con- 

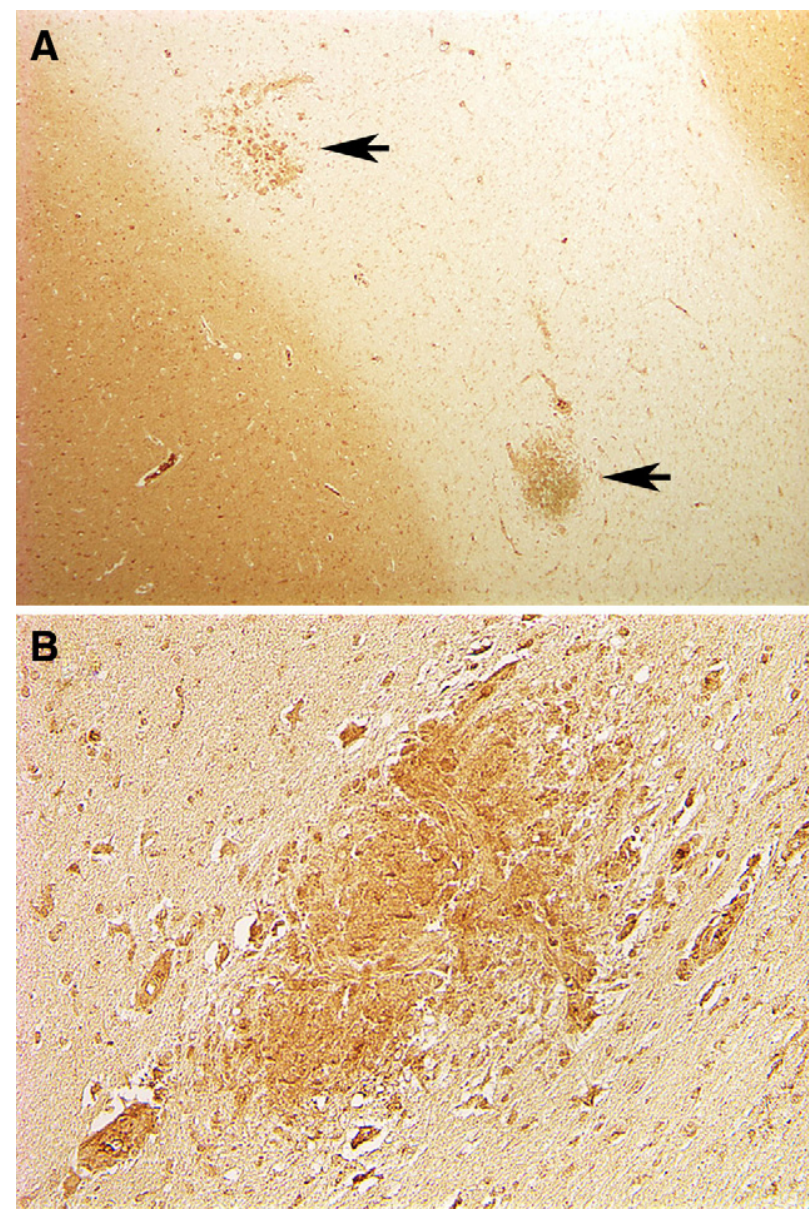

Figure 1. SP expression in brain of macaques with SIV encephalitis. A and B: Diffuse labeling of the gray matter and focal labeling of SIVE lesions in the white matter (arrows in A). The positive cells are morphologically compatible with perivascular macrophages and multinucleated giant cells typically found in SIVE lesions. Original magnification: $\times 5(\mathbf{A}) ; \times 10(\mathbf{B})$.

sistent with perivascular macrophages and multinucleated giant cells.

\section{NK1-R and SIV Infection in Vivo}

Localization of SIV-infected cells in brain was examined by in situ hybridization using riboprobes to detect viral RNA. This was combined with immunofluorescence for NK1-R to examine the relationship between expression of SP and its receptor in the context of SIV infection. The immunophenotype of infected cells and cells expressing NK1-R was then further characterized with multilabel immunofluorescence for a variety of cell type specific markers combined with in situ hybridization for SIV (Tables 2 and 3).

\section{In Normal and Nonencephalitic Brain}

In brain of normal, acutely SIV-infected animals and SIVinfected animals without SIVE, labeling of NK1-R revealed a basal level of expression limited to neurons and astrocytes (Figure 2). Double-label immunofluorescence using NK1-R and the astrocyte marker, GFAP, clearly showed diffuse low-level expression of NK1-R by astrocytes in normal con-
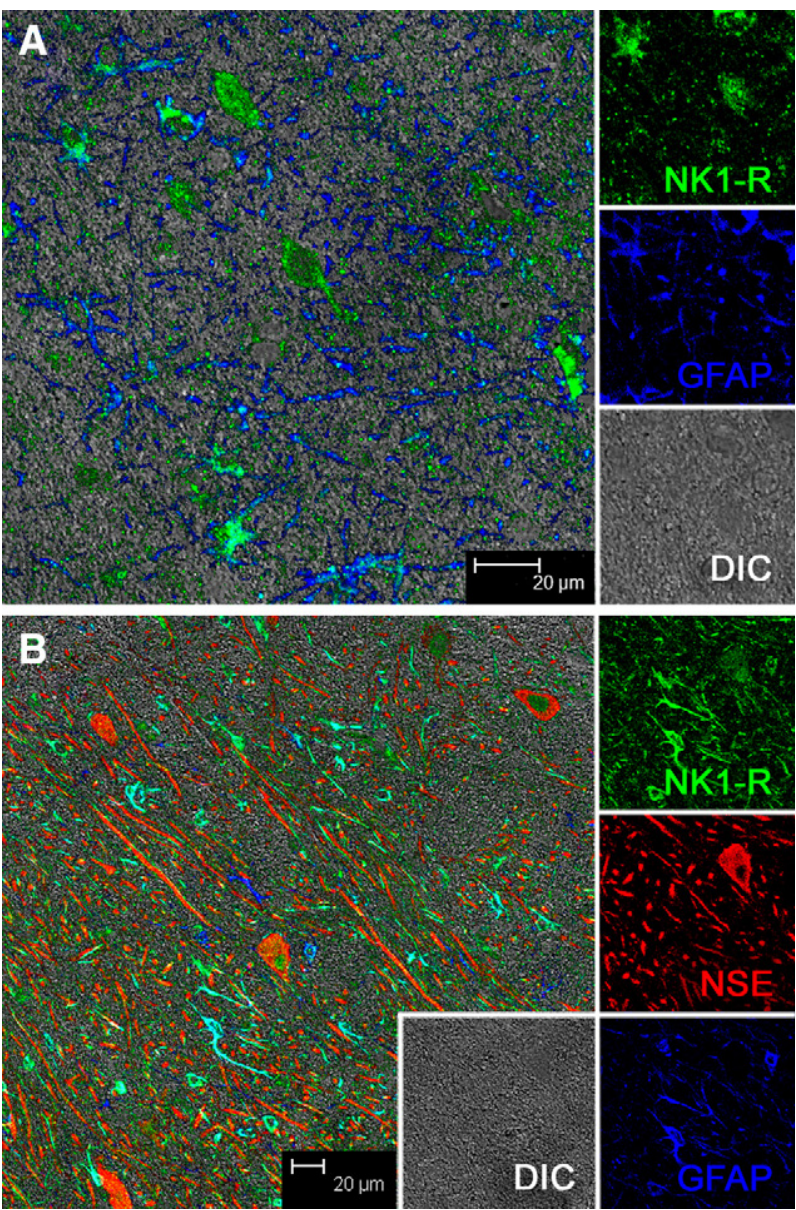

Figure 2. Multilabel confocal microscopy of NK1-R expression in normal brain Individual channels are shown on the right with a larger merged image on the left. A: Double-label confocal microscopy showing colocalization of NK1-R (with Alexa 488, green) and GFAP (with Alexa 633, blue), indicating that NK1-R is expressed by astrocytes in brains of normal animals. The differential interference contrast, DIC, is shown in gray. B: Triple-label confocal microscopy showing that NK1-R (green) is expressed by individual neurons (NSE with Alexa 568, red) and astrocytes (GFAP, blue) in brains of normal animals.

trols (Figure 2A). Additionally, triple-label immunofluorescence using NK1-R, GFAP, and a neuronal marker, NSE, demonstrate a similar basal level of NK1-R expression among neurons in normal brain (Figure 2B). No NK1-R expression was detected in immune cells in brains of any of the normal and non-encephalitic controls.

\section{In SIVE}

In contrast to normal and acutely infected animals without neurological disease, in animals with SIVE, the expression of NK1-R was very prominent and associated with SIVE lesions (Figure 3), which consist primarily of monocyte/ macrophages. To confirm that the cells expressing NK1-R were monocyte/macrophages, we examined NK1-R expression and CD163 (a macrophage marker) together (Figure $3 \mathrm{~A}$ ) and NK1-R expression on $\mathrm{CD}^{+} \mathrm{T}$ cells (Figure $3 \mathrm{~B}$ ). While rare, $T$ cells were present in SIVE lesions and do express NK1-R, however the vast majority of cells in the SIVE lesions were macrophages expressing CD163 and/or HAM56. Colocalization of NK1-R and CD163 is 

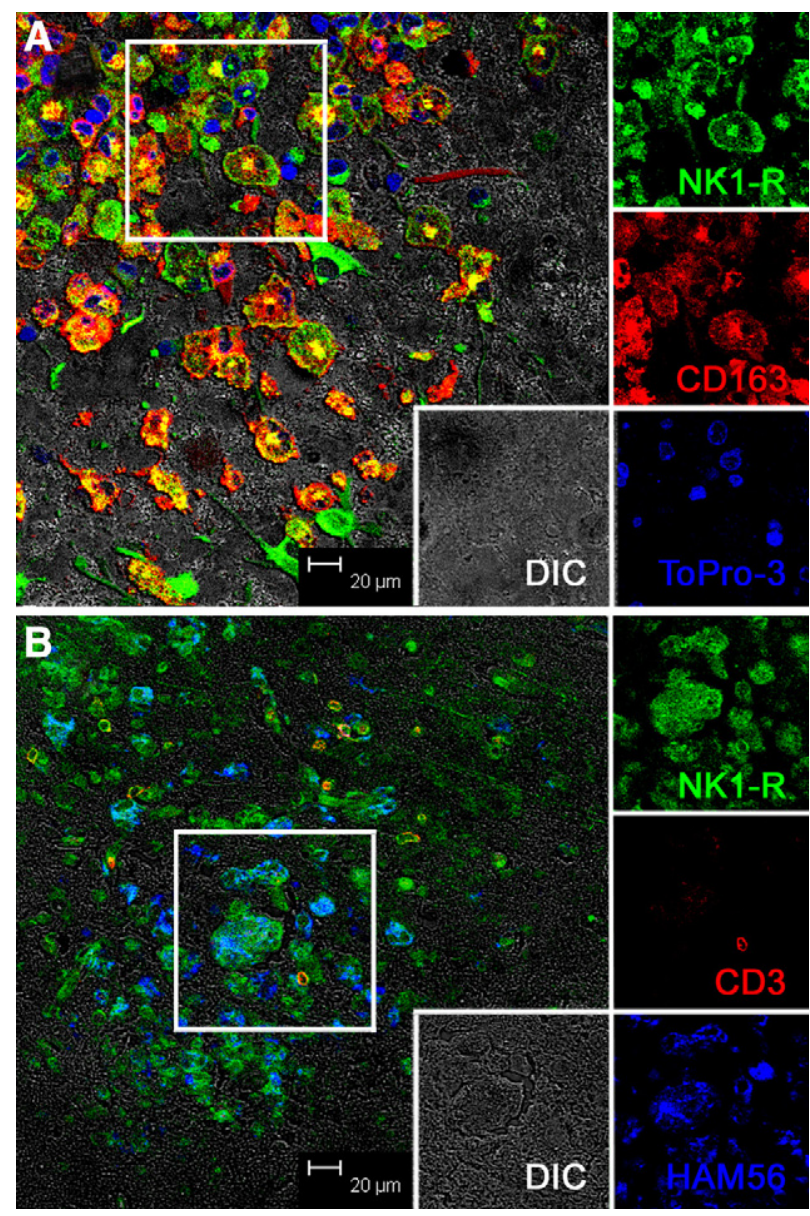

Figure 3. Multilabel confocal microscopy of NK1-R expression in SIVE brain. Individual channels are shown on the right with a larger merged image on the left. The individual channels are from the area indicated by a box in the larger image on the left. A: Triple-label confocal microscopy of NK1-R expression in SIVE brain showing NK1-R expression (green) by accumulated $\mathrm{CD} 163^{+}$(red) perivascular macrophages and multinucleated giant cells within an SIVE lesion. Note the colocalization of NK1-R and CD163 (yellow to orange) in many of the cells [NK1-R with Alexa 488, green; CD163 with Alexa 568, red; nuclei with To-pro3, blue, and differential interference contrast (DIC), gray]. B: Triple-label confocal microscopy showing a small number of T cells in SIVE lesions expressing NK1-R and numerous macrophages expressing NK1-R. The T cells appear yellow to orange due to colocalization of CD3 (red) and NK1-R (green), and the macrophages appear blue-green due to colocalization of HAM56 (blue) and NK1-R (green). [NK1-R with Alexa 488, green; CD3 with Alexa 568, red; HAM56 with Alexa 633, blue; and differential interference contrast (DIC), gray].

further shown at a higher magnification in Supplemental Figure S2 (at http://ajp.amjpathol.org). Expanding this analysis to include in situ hybridization for SIV, as well as detection of NK1-R, confirmed prior work that showed that the productively infected cells are CD163+ macrophages and extended it by showing that these cells also expressed NK1-R (Figure 4, A-C).

\section{SP Signaling via NK1-R Elicits Enhanced Cell Surface Receptor Expression of NK1-R and CCR5 through Receptor Cross Talk}

To determine whether NK1-R expression on monocytes was functionally regulated by SP during inflammation in SIV, we analyzed changes in expression of NK1-R and
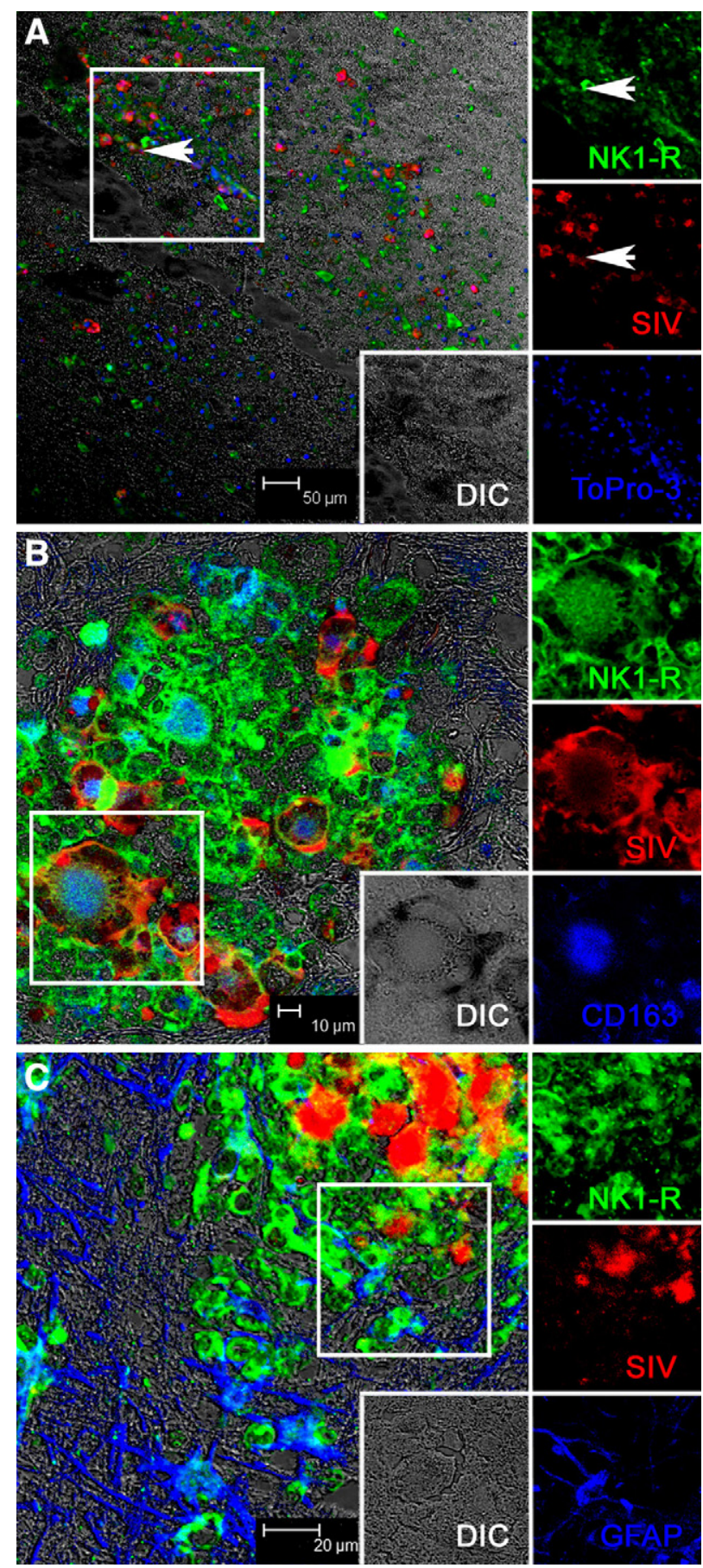

Figure 4. Multilabel confocal microscopy of NK1-R expression by SIV-infected cells in SIVE brain. Individual channels are shown on the right with a larger merged image on the left. The individual channels are from the area indicated by a box in the larger image on the left. A: Low-magnification triple-label confocal microscopy showing that NK1-R is expressed by SIV-infected cells at sites of inflammation. Arrowheads point out infected cells expressing NK1-R. [NK1-R with Alexa 488, green; SIV in situ hybridization with Permanent Red, red; nuclei with To-pro3, blue, and differential interference contrast (DIC), gray]. B: Higher magnification triple-label confocal microscopy showing that the SIV-infected cells (red) expressing NK1-R (green) within SIVE lesions are CD163 ${ }^{+}$macrophages (blue) and include multinucleated giant cells (MNGCs). [NK1-R with Alexa 488, green; SIV in situ hybridization with Permanent Red, red; CD163 with Alexa633, blue, and differential interference contrast (DIC), gray]. C: Triple-label confocal microscopy showing colocalization between NK1-R (green) and SIV in situ hybridization (red) resulting in yellow to orange labeling. NK1-R positive astrocytes are also visible at the margins of the SIVE lesion. [NK1-R with Alexa 488, green; SIV in situ hybridization with Permanent Red, red; GFAP (astrocytes) with Alexa633, blue, and differential interference contrast (DIC), gray]. 
A

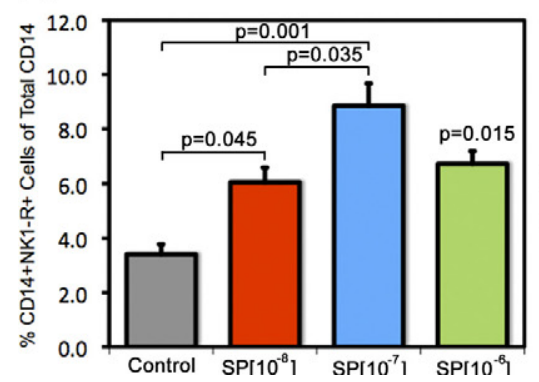

B

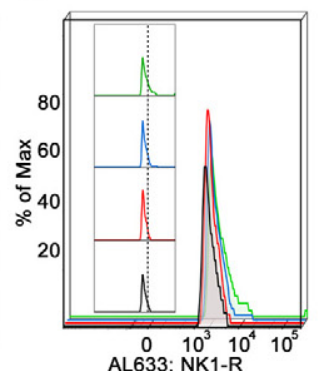

C

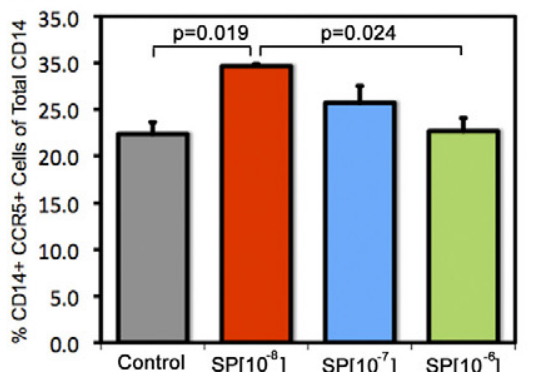

D

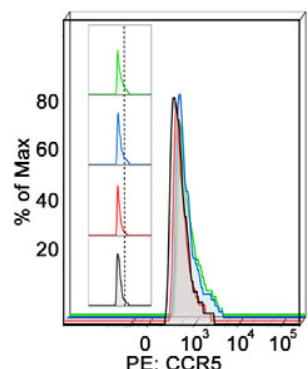

Figure 5. Changes in expression of NK1-R (A and $\mathbf{B})$ and CCR5 (C and $\mathbf{D})$ in response to treatment with SP. Bar graphs showing the percentage of monoyctes expressing NK1-R (A) or CCR5 $(\mathbf{C})$ in response to treatment of various concentrations of SP compared with control. Statistically significant differences are indicated with $P$ values in $\mathbf{A}$ and $\mathbf{C}$. In addition to group data showing changes in the population of monocytes expressing NK1-R or CCR5, representative graphs of fluorescence intensity of NK1-R expression per cell (B) and CCR5 expression per cell (D) are also provided from two animals. Note that there is a shift in fluorescence intensity. The colors used in the fluorescence intensity plots (B and $\mathbf{D}$ ) correspond to the concentrations of SP used in the bar graphs (A and $\mathbf{C}$ ).

CCR5 in response to treatment with varying concentrations of SP. Percentage of cells expressing either NK1-R or CCR5 as well as mean channel fluorescence intensity were examined and compared for each treatment group to distinguish a change in the percentage of cells expressing these receptors and/or a change in the amount/ density of receptor expression by such cells (Figure 5).

Treatment of monocytes with SP elicited changes in chemokine receptor expression for NK1-R in a bellshaped manner over a range of molar concentrations $\left(10^{-8}-10^{-6} \mathrm{M}\right.$; Figure $5 \mathrm{~A}$ ) with maximal expression of NK1-R occurring after treatment with $10^{-7} \mathrm{M}$ SP. The percentage of CD14 ${ }^{+}$monocytes expressing NK1-R was significantly increased compared with control at each concentration of SP used $\left(10^{-8}-10^{-6} \mathrm{M}\right)$. For CCR5, maximum expression on $\mathrm{CD}_{14^{+}}$monocytes followed treatment with $10^{-8} \mathrm{M} \mathrm{SP}$ and declined with increasing concentrations of SP (Figure $5 \mathrm{C}$ ).

In addition to looking at the percentage of $\mathrm{CD} 14^{+}$ monocytes expressing either NK1-R or CCR5 in response to SP, we also examined mean channel fluorescence intensity (MFI) to assess density of receptor expression on a per cell basis. As can be seen from the histograms, treatment with SP results in an increase in the number of cells expressing both receptors as described above, but also an increase in the fluorescence intensity of such cells. For NK1-R, the fluorescence intensity data appear quite similar to the percentage of cell surface expression of NK1-R on CD14 ${ }^{+}$, such that increased cellular expression of NK1-R with SP $\left(10^{-8} \mathrm{M}\right)$ treatment coincides with increased intensity of NK1-R expression by those cells as shown in Figure 5 B. Similarly, treatment with $\operatorname{SP}\left(10^{-7} \mathrm{M}\right)$ significantly enhances percent cellular expression as well as intensity of surface expression of NK1-R. Treatment with SP $\left(10^{-6} \mathrm{M}\right)$ results in a marked decrease in cell surface expression of NK1-R compared with that of treatment with SP $\left(10^{-7} \mathrm{M}\right)$, yet results in further increase in intensity of receptor expression on those cells clearly shown in Figure 5B. For CCR5 the MFI data suggest that the control has the lowest MCF followed by $10^{-8}$ with $10^{-7}$ and $10^{-6}$ having the highest $\mathrm{MFI}$, as shown in Figure 5D. This suggests that SP caused changes in the cellular density of CCR5/cell but not in the percentage of
$\mathrm{CD}_{14}{ }^{+}$cells expressing CCR5 except for the $10^{-8} \mathrm{M}$ treatment group.

These results indicate that SP is capable of modulating expression of both NK1-R and CCR5 in macaque monocytes, supporting SP's role as a proinflammatory mediator on monocytes. Moreover, SP treatment resulted in increased expression of CCR5, which is the major coreceptor for HIV and SIV. These results suggest that CCR5 and NK1-R signaling pathways are capable of interacting and could potentiate SIV or HIV infection of monocytes/ macrophages, which would be particularly important in the neuropathogenesis of AIDS.

\section{SP Induces Chemotactic Signaling in Monocytes and Potentiates CCR5-Mediated Chemotaxis}

The functional role of SP signaling through NK1-R on monocyte chemotaxis was examined by implementing a transmigration assay. We assessed the ability of SP to induce monocyte chemotaxis in comparison with that of CCL5, a known chemokine as well as the ligand for CCR5. Additionally, possible cross talk between the signaling pathways of NK1-R and CCR5 were examined by assessing the ability of SP and CCL5 combined to induce monocyte chemotaxis. In addition to comparing the effects of CCL5 and SP alone and together on chemotaxis we also examined the impact of SP pretreatment on these responses (Figure 6, A-C). Resulting numbers of migrated cells from these experiments were recorded (see Supplemental Table 2 at http://ajp.amjpathol.org) as described in the Materials and Methods and reported in Figure $6 \mathrm{C}$ as a percentage increase in the number of migrated monocytes over the control chemotactic media (media alone).

In the experimental group pretreated with media alone, there was enhanced chemotaxis in response to SP [8057 \pm 946 cells, $P<0.005$ (mean \pm SE, $n=8$ )], CCL5 $[8291 \pm 1014$ cells, $P<0.005$ (mean $\pm \mathrm{SE}, n=8)]$, and $\mathrm{SP}+\mathrm{CCL} 5[12,456 \pm 552$ cells, $P<0.005$ (mean \pm SE, $n=8)$, as compared with media alone as the chemoattractant. No statistical difference between the chemotac- 


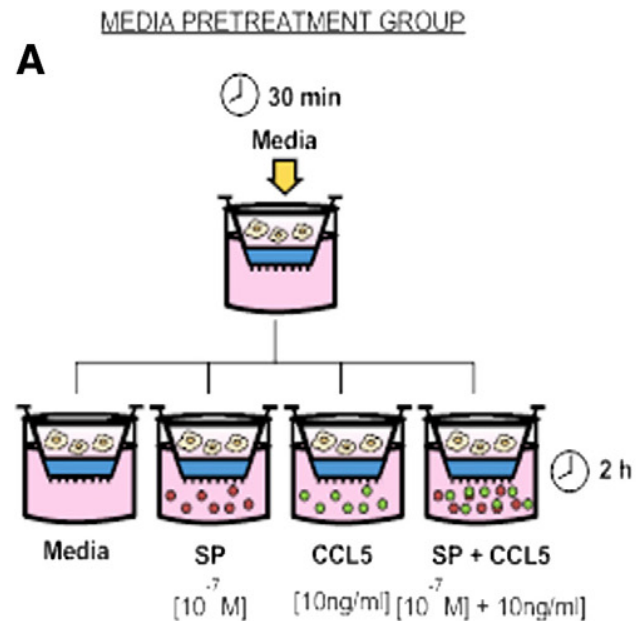

B

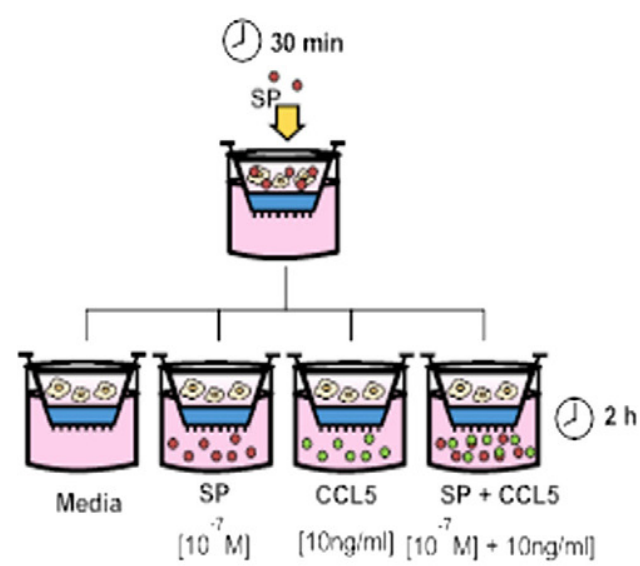

\section{C $\%$ Increase Over Media in Number of Migrated Monocytes}

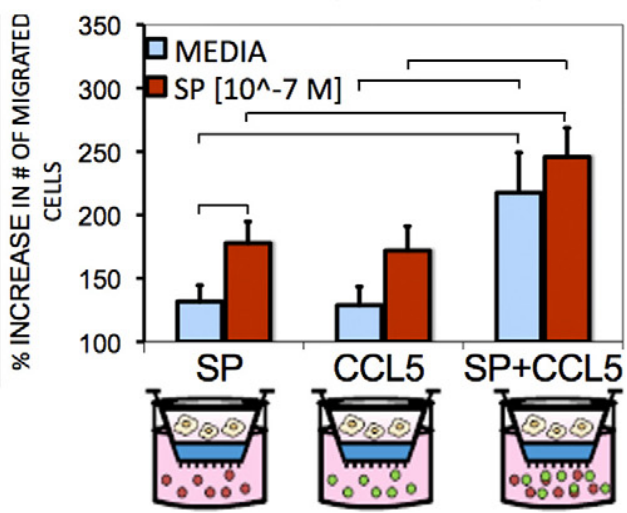

Figure 6. Schematic depicting the experimental design for the media (A) and SP (B) pretreatment groups in the chemotaxis assay. Following pretreatment, cells were placed in the upper well and their response to chemotactic stimuli (SP, CCL5, SP + CCL5, or media alone) was assessed C: Bar graph of the percent increase in the number of migrated monocytes over that observed using media alone as the chemoattractant. Blue bars represent no pretreatment (media alone), while red bars represent results following pretreatment with $10^{-7} \mathrm{M} \mathrm{SP}$. All of the chemoattractant stimuli (SP, CCL5, SP + CCL5) resulted in significant increases in chemotaxis compared with control media $(P$ values $<0.05)$ regardless of pretreatment. Furthermore, SP + CCL5 as the chemoattractant resulted in greater chemotaxis than either CCL5 or SP alone regardless of pretreatment. Pretreatment with SP did significantly enhance migration to SP as a chemoattractant. $P<0.05$ for comparisons between groups indicated by horizontal bars in the figure. tic response elicited by SP or CCL5 was observed, indicating that SP mediates a chemotactic response as well as CCL5.

This same pattern of chemotactic responses were observed when cells were pretreated with SP $\left(10^{-7} \mathrm{M}\right)$; Enhanced chemotaxis compared with media alone as the chemoattractant was observed in response to SP $[12,806 \pm 2098, P<0.005$ (mean $\pm \mathrm{SE}, n=8$ )], CCL5 $[11,950 \pm 1476, P<0.005$ (mean $\pm \mathrm{SE}, n=8)]$, and $\mathrm{SP}+\mathrm{CCL} 5[18,217 \pm 2909, P<0.005$ (mean $\pm \mathrm{SE}, n=$ 8)], and there was no difference between chemotactic responses elicited by SP or CCL5.

When SP and CCL5 were combined as chemoattractants (irrespective of pretreatment condition), a statistically significant increase in the percentage of monocyte chemotaxis over baseline compared with either SP or CCL5 alone was observed $(P<0.05)$ as illustrated in Figure 6.

Comparison of results from both pretreatment conditions (media versus SP alone) showed that pretreatment with SP significantly enhanced migration to SP as a chemoattractant. SP pretreatment also increased CCL5 mediated chemotaxis; however, the results were not statistically significant. These findings indicate that SP can sensitize/prime monoyctes for migration in addition to serving as a chemoattractant. Collectively, these data indicate that SP/NK1-R and CCL5/CCR5 signaling pathways interact such that the downstream effects of such signaling (eg, chemotaxis) are potentiated.

\section{Discussion}

SP, an 11-aa neuropeptide tachykinin, is elevated in the serum of men and women with HIV. ${ }^{9} \mathrm{SP}$ is an important neuroimmune modulator, which has proinflammatory activities in infections including HIV. Our studies in human cells have demonstrated that SP enhances HIV infection of monocyte-tropic strains of HIV. ${ }^{15,22}$ Further, NK1-R antagonists, such as CP96345 and aprepitant, inhibited M-tropic HIV by down-regulating the chemokine receptor CCR5 ${ }^{15,22,29}$

In our investigation, we examined the expression of SP and its cognate receptor NK1-R in normal and SIV-infected rhesus macaques with and without SIV encephalitis (SIVE). We demonstrate that SP in normal and SIVinfected macaques without SIV is expressed in neurons and astrocytes, primarily in the gray matter as previously reported. ${ }^{27,28}$ In macaques with SIVE, SP immunoreactivity was demonstrated in the white matter in association with SIVE lesions in cells morphologically compatible with perivascular macrophages and multinucleated giant cells. The findings are consistent with our observed expression of NK1-R in the cingulate cortex of individuals who demonstrated HIV encephalitis at postmortem in human brains. ${ }^{9}$ Immunohistologic studies in acute SIV infection and SIV-infected animals without SIVE demonstrated NK1-R expression in neurons and astrocytes. In SIVE, however, the expression of NK1-R was much more prominent than in normal animals or SIV-infected animals without SIVE and was observed in cells of the immune 
system, in particular monocyte-macrophages in perivascular cuffs typical of SIVE. Further, most of the macrophages and MNGCs in these lesions expressed NK1-R. Moreover, all of the cells in SIVE lesions infected with SIV also expressed NK1-R. These observations further support the concept that in SIV infection as well as in HIV, SP and its receptor NK1-R have important roles in the regulation of proinflammatory signaling.

Our recent studies ${ }^{26}$ demonstrate that treatment of human monocytes with SP leads to activation of the truncated form of NK1-R, modulating CCR5-mediated cellular responses. In particular, SP-enhanced CCR5 ligand CCL5 elicited calcium mobilization and enhanced CCL5mediated chemotaxis. ${ }^{26}$ The amino acid sequence in the truncated NK1-R isoform overlaps perfectly with the sequence of the first 311 amino acid residues in the full length receptor, but it lacks 97 amino acids that constitute most of the intracellular domain at the $\mathrm{C}$ terminus. ${ }^{8,30}$ This truncated receptor isoform may co-exist with the full-length receptor, and it has been shown to have lower affinity for SP, as compared with its full-length counterpart. ${ }^{30}$ In the present study, using a flow cytometry approach, we have demonstrated that in rhesus macaques, the treatment of monocytes with SP elicited changes in cell-surface expression of CCR5 and NK1-R. CCR5 expression was significantly increased by SP treatment at concentrations of $10^{-8} \mathrm{M}$; however, a concentration of SP as high as $10^{-6} \mathrm{M}$ did not significantly alter the expression of CCR5 as compared with control cells. Interestingly, the expression of NK1-R was increased when SP was added to cells at concentrations of $10^{-8} \mathrm{M}, 10^{-7} \mathrm{M}$, and $10^{-6} \mathrm{M}$. However, NK1-R expression was significantly higher when cells were treated with SP at a concentration of $10^{-7} \mathrm{M}$ compared with SP treatment at concentrations of $10^{-8} \mathrm{M}$ and $10^{-6} \mathrm{M}$. These observations demonstrate that complex mechanisms might be governing the signaling pathways downstream of NK1-R. The difference between the dose-response curves of SP for inducing CCR5 and NK1-R expression might be related to the process of internalization that is part of the termination of the signaling mechanism of NK1-R. ${ }^{31,32}$ It has been demonstrated that on binding of SP to NK1-R, the intracellular signaling triggered by NK1-R activation is promptly terminated by a mechanism that involves $\beta$-arrestin recruitment and receptor internalization. Thus, it is expected that full activation of the NK1-R with high concentrations of SP will result in decreased NK1-R expression, because the receptor will be internalized, and thus it becomes inaccessible to the NK1-R antibodies that were used for flow cytometry staining. This is not the case for the CCR5 receptor, which is not internalized following monocyte activation with SP. Thus, our quantitative flow cytometric analysis demonstrates that SP functionally regulates NK1-R and CCR5 expression in macaque monocytes. Further, pretreatment with SP enhanced SP mediated chemotaxis. These findings are consistent with the findings of Chernova et al, showing that SP-mediated signaling in the presence of CCR5 potentiates CCR5 signaling in human monocytes. Our results demonstrate the involvement of SP/NK1-R signaling on CCL5 elicited chemotaxis. The enhancement of SP-mediated chemo- taxis in the presence of CCL5 suggests that there is a cross talk between the intracellular signaling pathways initiated by CCR5 and NK1-R. Our findings further support the role of SP as a proinflammatory mediator of monocyte activation and chemotaxis. Taken together, these data support the concept that SP has a significant role in augmenting cellular trafficking in the central nervous system at the blood-brain barrier, contributing to neurological disease in SIV and in SIVE.

\section{Acknowledgment}

We thank Robin Rodriguez for assistance with the figures.

\section{References}

1. Ho W-Z, Douglas SD: Substance P and neurokinin-1 receptor modulation of HIV. J Neuroimmunol 2004, 157:48-55

2. Leserman J: HIV disease progression: depression, stress, and possible mechanisms. Biol Psychiatry 2003, 54:295-306

3. Williams KC, Hickey WF: Central Nervous System Damage, Monocytes and Macrophages, and Neurological Disorders in AIDS, Annu Rev Neurosci 2002, 25:537-562

4. Gonzalez-Scarano F, Martin-Garcia J: The neuropathogenesis of AIDS. Nat Rev Immunol 2005, 5:69-81

5. Navia B, Cho E, Petito C, Price R: The AIDS dementia complex: II Neuropathology. Ann Neurol 1986, 19:517-524

6. Navia B, Cho E, Petito C, Price R: The AIDS dementia complex: I Clinical Features. Ann Neurol 1986, 19:525-535

7. Price R, Brew B, Sidtis J, Rosenblum M, Scheck A, Cleary P: The brain in AIDS: central nervous system HIV-1 infection and AIDS dementia complex. Science 1988, 239:586-592

8. Tuluc F, Lai JP, Kilpatrick LE, Evans DL, Douglas SD: Neurokinin 1 receptor isoforms and the control of innate immunity. Trends Immunol 2009, 30:271-276

9. Douglas SD, Lai J-P, Tuluc F, Schwartz L, Kilpatrick LE: Neurokinin-1 receptor expression and function in human macrophages and brain Ann NY Acad Sci 2008, 1144:90-96

10. Masliah E, Ge N, Morey M, DeTeresa R, Terry R, Wiley C: Cortical dendritic pathology in human immunodeficiency virus encephalitis. Lab Invest 1992, 66:285-291

11. Crews L, Lentz MR, GonzÃ̈lez RG, Fox HS, Masliah E: Neuronal injury in simian immunodeficiency virus and other animal models of neuroAIDS. J Neurovirol 2008, 14:327-339

12. Annunziata P, Cioni C, Toneatto S, Paccagnini E: HIV-1 gp120 increases the permeability of rat brain endothelium cultures by a mechanism involving substance P. AIDS 1998, 12:2377-2385

13. Li Y, Douglas SD, Song L, Sun S, Ho W-Z: Substance P enhances HIV-1 replication in latently infected human immune cells. J Neuroimmunol 2001, 121:67-75

14. Annunziata $P$ : Blood-brain barrier changes during invasion of the central nervous system by HIV-1. J Neurol 2003, 250:901-906

15. Lai J-P, Ho W-Z, Zhan G-X, Yi Y, Collman RG, Douglas SD: Substance $P$ antagonist (CP-96,345) inhibits HIV-1 replication in human mononuclear phagocytes. Proc Natl Acad Sci USA 2001, 98:3970-3975

16. Ho W-Z, Lai J-P, Li Y, Douglas SD: HIV enhances substance P expression in human immune cells. FASEB J 2002, 16:616-618

17. Ho W, Cnaan A, Li Y, Zhao H, Lee H, Song L, Douglas S: Substance $P$ modulates human immunodeficency virus replication in human peripheral blood monocyte-derived macrophages. AIDS Res Hum Retroviruses 1996, 12:195-198

18. Annunziata P, Cioni C, Santonini R, Paccagnini E: Substance P antagonist blocks leakage and reduces activation of cytokine-stimulated rat brain endothelium. J Neuroimmunol 2002, 131:41-49

19. O'Connor TM, O'Connell J, O'Brien DI, Goode T, Bredin CP, Shanahan $\mathrm{F}$ : The role of substance $\mathrm{P}$ in inflammatory disease. J Cell Physiol 2004, 201:167-180

20. Derocq J-M, Ségui M, Blazy C, Emonds-Alt X, Le Fur G, Brelière J-C Casellas $P$ : Effect of substance $P$ on cytokine production by human 
astrocytic cells and blood mononuclear cells: characterization of novel tachykinin receptor antagonists. FEBS Lett 1996, 399:321-325

21. Lai J-P, Ho W-Z, Yang J-H, Wang X, Song L, Douglas SD: A nonpeptide substance $P$ antagonist down-regulates SP mRNA expression in human mononuclear phagocytes. J Neuroimmunol 2002, 128:101-108

22. Wang $X$, Douglas S, Lai J-P, Tuluc F, Tebas P, Ho W-Z: Neurokinin-1 Receptor Antagonist (Aprepitant) Inhibits Drug-Resistant HIV-1 Infection of Macrophages in vitro. J Neuroimmune Pharmacol 2007, 2:42-48

23. Wang X, Douglas S, Song L, Wang Y-J, Ho W-Z: Neurokinin-1 receptor antagonist (aprepitant) suppresses HIV-1 infection of microglia/ macrophages. J Neuroimmune Pharmacol 2008, 3:257-264

24. Kramer MS, Cutler N, Feighner J, Shrivastava R, Carman J, Sramek JJ, Reines SA, Liu G, Snavely D, Wyatt-Knowles E, Hale JJ, Mills SG, MacCoss M, Swain CJ, Harrison T, Hill RG, Hefti F, Scolnick EM, Cascieri MA, Chicchi GG, Sadowski S, Williams AR, Hewson L, Smith D, Carlson EJ, Hargreaves RJ, Rupniak NM: Distinct mechanism for antidepressant activity by blockade of central substance $P$ receptors. Science 1998, 281:1640-1645

25. Borda JT, Alvarez X, Mohan M, Hasegawa A, Bernardino A, Jean S, Aye P, Lackner AA: CD163, a marker of perivascular macrophages, is up-regulated by microglia in simian immunodeficiency virus encephalitis after haptoglobin-hemoglobin complex stimulation and is suggestive of breakdown of the blood-brain barrier. Am J Pathol 2008, 172:725-737

26. Chernova I, Lai J-P, Li H, Schwartz L, Tuluc F, Korchak HM, Douglas SD, Kilpatrick LE: Substance P (SP) enhances CCL5-induced chemotaxis and intracellular signaling in human monocytes, which express the truncated neurokinin-1 receptor (NK1R). J Leukoc Biol 2009, 85:154-164

27. Pernow B: Substance P. Pharmacol Rev 1983, 35:85-141

28. Nakanishi S: Substance $P$ precursor and kininogen: their structures, gene organizations, and regulation. Physiol Rev 1987, 67:1117-1142

29. Lai JP, Ho WZ, Kilpatrick LE, Wang X, Tuluc F, Korchak HM, Douglas SD: Full-length and truncated neurokinin-1 receptor expression and function during monocyte/macrophage differentiation. Proc Nat Acad Sci U S A 2006, 103:7771-7776

30. Fong TM, Anderson SA, Yu H, Huang RR, Strader CD: Differential activation of intracellular effector by two isoforms of human neurokinin-1 receptor. Mol Pharmacol 1992, 41:24-30

31. Schmidlin F, Roosterman D, Bunnett NW: The third intracellular loop and carboxyl tail of neurokinin 1 and 3 receptors determine interactions with \{beta\}-arrestins. Am J Physiol Cell Physiol 2003, 285: C945-C958

32. Cottrell GS, Padilla BE, Amadesi S, Poole DP, Murphy JE, Hardt M Roosterman D, Steinhoff M, Bunnett NW: Endosomal endothelin-converting enzyme-1. J Biol Chem 2009, 284:22411-22425 